\title{
Performance Analysis of a Full-Duplex Cooperative Diversity Scheme with Partial Channel Knowledge at the Cooperating Nodes
}

\author{
Renato Machado, Bartolomeu F. Uchôa-Filho, and Tolga M. Duman
}

\begin{abstract}
We propose a cooperative diversity scheme for a communication system consisting of $N_{T}$ cooperating nodes that receive $\left\lceil\log _{2}\left(N_{T}\right)\right\rceil$ feedback bits from the destination node on the channel state information. This information is used appropriately to obtain cooperative diversity and signal-to-noise ratio (SNR) gains. A simple linear detector and an interference cancelation detector are proposed. It is shown that their error rates are quite close to that of the maximum likelihood detector. An upper bound for the average bit error probability for binary phase-shift keying (BPSK) modulation over a Rayleigh fading channel is derived. In addition, through computer simulations, it is verified that the proposed scheme offers a good error performance when the inter-user channel SNR is high or when the inter-user channel has a well-defined line-of-sight component. In other words, the new scheme becomes interesting when the cooperating nodes are close to each other.
\end{abstract}

Index Terms-Cooperative diversity, node selection, limited feedback, power allocation, space-time codes.

\section{INTRODUCTION}

$\mathbf{M}$ ULTIPLE-ANTENNA techniques are quite attractive for deployment in cellular applications at base stations and have already been included in the 3 rd generation wireless standards. Unfortunately, in some wireless scenarios, transmitters are very small in size and cannot support the use of multiple antennas. To address this limitation, cooperative diversity schemes have been proposed [1], [2]. The basic idea behind cooperative diversity rests on the observation that, in a wireless environment, the signal transmitted by a source node is "overheard" by other nodes, which can be viewed as "partners". The source and its partners can jointly process and transmit their information, creating a "virtual antenna array" although each one of them is equipped with only one antenna.

Since the work of Sendonaris et al. [1], [2], the interest in cooperative communications has grown considerably. Sendonaris et al. have proposed algorithms for cooperation in a code-division multiple-access (CDMA) framework, where each mobile decodes and relays certain number of bits received from its partner. In [3], it is shown that both amplify-andforward and adaptive methods achieve diversity order of

Manuscript received September 22, 2009; revised May 28, 2010. R. Machado is with the Communications and Networking Systems Research Group, CTISM, Federal University of Santa Maria, Santa Maria, RS, 97105-900, BRAZIL. E-mail: renatomachado@redes.ufsm.br. B. F. Uchôa-Filho is with the Communications Research Group, Department of Electrical Engineering, Federal University of Santa Catarina, Florianópolis, SC, 88040-900, BRAZIL. E-mail: uchoa@eel.ufsc.br. T. M. Duman is with the School of Electrical, Computer and Energy Engineering, Arizona State University, Tempe, AZ, 85287-5706, U.S.A. E-mail: duman@asu.edu.

This paper was presented in part at the IEEE International Conference on Communications (ICC’08), Beijing, China, May 2008. two for two-user cooperation. Laneman et al. [4] suggest "conventional" orthogonal space-time block coding (STBC) (originally proposed for coding across co-located antennas in [5], [6]) for practical implementation of user cooperation in a "distributed" fashion.

A cooperative transmit diversity scheme for two cooperating nodes based on superposition modulation and multiuser detection was proposed by Larsson and Vojcic [7]. In that scheme, two cooperating nodes act as relays for one another. When one cooperating node acts as the relay for the other node, it simultaneously transmits its own data and the data for which it acts as relay, using superposition modulation. A soft-MAP-based multiuser detection is used at the destination node in order to recover the two data streams. It is shown that this scheme outperforms the classical "decode-and-forward" methods [1]-[4].

A wireless communication system can obtain significant performance improvements when the channel state information (CSI) is available at the transmitters [8]-[14]. In [11], [12] CSI is exploited in the context of cooperation. Ahmed et al. [13] have considered practical methods to approach the theoretical performance limits of the fading relay channel under different assumptions of channel knowledge at the transmitter for the typical relay scenario (i.e., source-relay-destination). In [14], the authors propose a cooperative diversity scheme called opportunistic relaying in which the "best" relay among $M$ candidates is selected for cooperation between source and destination. Although the authors in [14] consider simultaneous transmissions by the "best" relay and the source, in their analysis they allow only one transmission each time interval.

In the current literature (including the works cited above), typically, the cooperating nodes either acting as relays only or acting both as data sources and relays use some form of orthogonal communication such as CDMA or time-division multiple-access (TDMA). In other words, the cooperating nodes are assumed to be half-duplex. Full-duplex relays, in contrast, are able to transmit and receive signals simultaneously and the destination receives superposition of the direct and relayed signals. Although full-duplex operation must rely on perfect electromagnetic isolation and/or perfect echo cancelation between the transmit and receive paths, which is technologically more difficult to achieve, full-duplex relays offer higher capacities over half duplex relays as they avoid additional use of time slots. Full-duplex relays have recently been considered to exploit this additional degree of freedom [15]-[18]. In particular, the authors in [18] present an inter- 
esting interference cancelation method based on precoding to realize a new full-duplex relay system.

In this paper, we assume that the cooperating nodes can operate in full-duplex transmission mode, i.e., whenever employed these nodes can transmit signals to the destination node while receiving signals from some other cooperating node. In turn, the destination node receives superimposed signals. We propose and analyze a cooperative diversity scheme for $N_{T} \geq 2$ full-duplex transmit nodes and $N_{D}=1$ destination node, all of which equipped with a single antenna. The channels among the transmit nodes (inter-user channels) are assumed to be independent of the channels from the transmit nodes to the destination node (forward channels), and the forward channels are assumed to be mutually independent.

In the proposed scheme, the transmit nodes receive $\left\lceil\log _{2}\left(N_{T}\right)\right\rceil$ bits of CSI before any data transmission begins. This corresponds to the number of bits necessary to indicate which forward channel is the best. This CSI information can be made available to the cooperating nodes either via a feedback channel or, more interestingly, by some distributed method such as the one based on local measurements of the instantaneous channel conditions presented in [14]. To keep the explanation simpler, we consider that the scenario that CSI is obtained through a feedback channel. Due to the distributed nature of this cooperative system, we cannot simply use selection combining by transmitting all symbols from the same source node, as in a centralized multiple-input singleoutput (MISO) system, since before the transmission starts each symbol is only available at its respective node. We should use for the transmission of the other symbols some small amount of power, just enough to make it sure that the symbols are overheard by the cooperating node having the strongest forward channel.

In a sense, the proposed scheme combines features of opportunistic relaying proposed in [14] and the scheme based on superposition modulation proposed in [7]. However, there are distinct features that make the cooperative diversity scheme proposed herein novel. First of all, the schemes in [7], [14] have been designed for half-duplex cooperative systems, while herein we consider full-duplex cooperating nodes. This fundamental difference naturally lends itself to different transmission protocols as well as different detection methods, not to mention the fact that in full-duplex cooperation systems, broadcasting and cooperation need not be performed at separate times. We also point out that while superposition in [7] is accomplished by the cooperating node before transmission, in our scheme it is a result of the simultaneous transmissions of data from two different cooperating nodes (one of which is the "best" node). In [7], there is no CSI at the cooperating nodes, and both nodes transmit at full power. Moreover, the scheme in [7] does not scale easily to a larger number of cooperating nodes. The detectors at the cooperating nodes and at the destination node would become more complex and error performance would degrade due to the increased interference level. In contrast, our proposed scheme suffers no performance degradation as the number of cooperating nodes increases, since the maximum number of simultaneous transmissions is kept to two at all times. Finally, in [14], there is only one source node, while all the other cooperating nodes act as relay only; hence, the situation is completely different from the one we consider in this paper.

The paper is organized as follows. In the next section, the system model is given. The proposed cooperative diversity scheme is described in Section III. In Section IV, we present the error performance analysis for the proposed scheme. We will observe that the well-known multiple access channel with interference model arises. A major difference of this work with the existing literature is that while the interfering symbol is transmitted through the (well-known) Rayleigh channel, the symbol of interest is transmitted through a channel whose statistical model is based on order statistics. In Section V, simulation results are presented. Finally, in Section VI, we present our conclusions and final comments.

\section{SYSTEM MODEL}

The full-duplex wireless communication system with cooperation considered in this paper is shown in Figure 1.

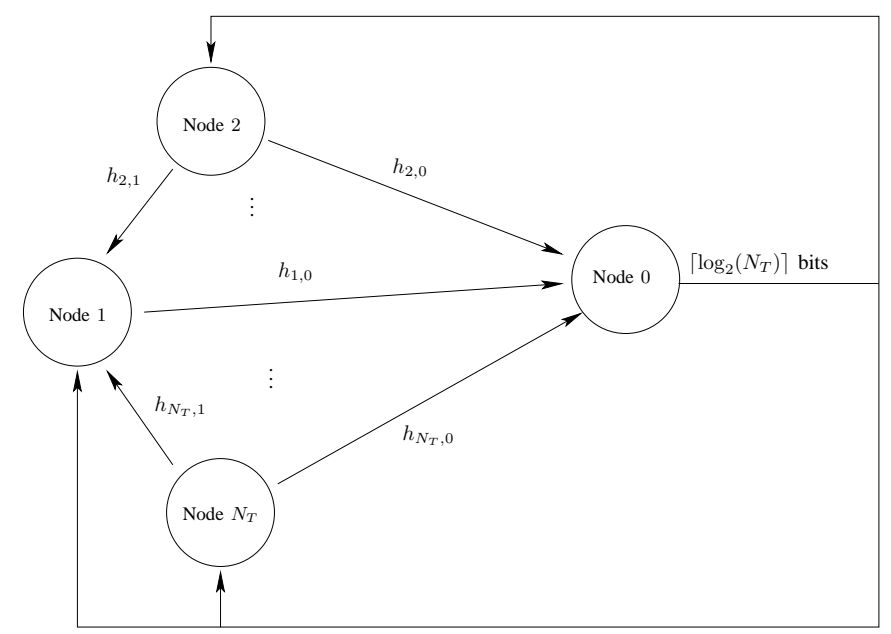

Fig. 1. Block diagram of the proposed cooperative scheme.

The scheme consists of $N_{T} \geq 2$ transmit nodes (called cooperating nodes) and $N_{D}=1$ receive (destination) node, all of them equipped with a single antenna. The forward channels are assumed to be Rayleigh fading channels. The signals received by the destination node are contaminated with additive white Gaussian noise (AWGN). The transmit nodes, named node 1 , node $2, \ldots$, node $N_{T}$, send their own information to the destination node, named node 0 , and also act as relays. The fading coefficients $h_{i, 0}$ associated with the channels from node $i$ to node $0, i=1,2, \ldots, N_{T}$, are modeled as independent zero mean circularly symmetric complex Gaussian random variables with variance $1 / 2$ per dimension. As we will see, the performance analysis carried out in Section IV is essentially independent of the particular statistical model assumed for the inter-user channels, as these are statistically independent of the forward channels (see Eq. (20)). Since the cooperating nodes are assumed to be close to each other, we model these channels as having a line-ofsight. The fading coefficients $h_{i, j}$, for $i, j=1,2, \ldots, N_{T}$ with $i \neq j$, are assumed non-zero mean complex Gaussian random 
variables with equal variances for each dimension, i.e., we assume Rician fading for the inter-user channels. All fading coefficients are assumed constant during the transmission of a block of $N_{T}$ consecutive symbols, changing randomly from one block to the next.

It is assumed that 1) the forward channel fading coefficients are known at the receiver, 2) when the cooperative node is in the full-duplex mode it can detect (possibly erroneously) the symbols coming from the other cooperative node, by knowing the corresponding inter-user channel fading coefficient, and 3) an error- and delay-free feedback channel is available through which $\left\lceil\log _{2}\left(N_{T}\right)\right\rceil$ bits can be sent towards the transmit nodes. Moreover, it is assumed that the feedback information arrives at the transmit nodes before any data transmission takes place. In other words, the feedback bits may be used to adapt the transmission to the instantaneous channel conditions right from the first symbol period.

\section{Proposed Cooperative Diversity Scheme With FEEDBACK CHANNEL}

In this section, the proposed cooperative diversity scheme is presented for the communication system whose model was described in the previous section. In the proposed scheme, at most two nodes transmit simultaneously in a given symbol period. Let $P_{+}$and $P_{-}$(with $P_{+}>P_{-}$) denote the transmit powers allocated to the strongest transmit node and to the other cooperating node, respectively, where the power constraint $P_{+}+P_{-}=P$ is enforced, $P$ being the total transmit power. For simplicity, we consider a two-level power allocation, say levels $\alpha$ and $P-\alpha$, where $\alpha$ can be optimized off-line and remains fixed at all times. We will see later on in this paper that $P_{+} \gg P_{-}$is a reasonable assumption.

Without loss of generality, for the purpose of analysis, we assume in the remainder of this paper that node 1 has the strongest forward channel, in other words, $\left|h_{1,0}\right|^{2}=$ $\max _{i}\left|h_{i, 0}\right|^{2}$, where $|\cdot|$ denotes absolute value. However, due to channel variations over time, each cooperating user will have the strongest forward channel with the same probability. Consequently, the proposed scheme will not incur battery shortage for any user in particular.

\section{A. The Transmitter}

Let $s_{1}, s_{2}, \ldots, s_{N_{T}}$ be data symbols from node 1 , node $2, \ldots$, node $N_{T}$, respectively, all of them belonging to a signal constellation with unit average energy. Assuming that $\left|h_{1,0}\right|^{2}=\max _{i}\left|h_{i, 0}\right|^{2}, i=1, \ldots, N_{T}$, more power should be allocated to node 1 , and the transmission in our cooperative diversity scheme is as described in Table I. First, node 1 transmits its own symbol $s_{1}$ with almost full power. At the same time, some other node say node 2 transmits its symbol $s_{2}$ with a lower power. The idea is that node 1 overhears $s_{2}$ but $s_{2}$ causes only a small amount of interference at the destination. In the next time slot, node 1 transmits the detected symbol $\tilde{s}_{2}$ with almost full power while at the same time some other node say node 3 transmits its symbol $s_{3}$ with low power. The process continues until node 1 transmits the detected symbol of the last node to transmit with low power, say node $N_{T}$.
At this time, without any other interfering transmission, node 1 transmits $\tilde{s}_{N_{T}}$ with full power. The decisions made at the

TABLE I

TRANSMISSION SCHEME

\begin{tabular}{ccccccc}
\hline & When $\left|h_{1,0}\right|$ & $=\max _{i}\left|h_{i, 0}\right|$ & \multicolumn{2}{c}{$i=1,2, \ldots, N_{T}$} \\
\hline \hline Node & Time 1 & Time 2 & $\ldots$ & Time $N_{T}-1$ & Time $N_{T}$ \\
\hline 1 & $\sqrt{P_{+}} s_{1}$ & $\sqrt{P_{+}} \tilde{s}_{2}$ & $\ldots$ & $\sqrt{P_{+} \tilde{s}_{N_{T}-1}}$ & $\sqrt{P} \tilde{s}_{N_{T}}$ \\
\hline 2 & $\sqrt{P_{-} s_{2}}$ & 0 & $\ldots$ & 0 & 0 \\
\hline 3 & 0 & $\sqrt{P_{-} s_{3}}$ & $\ldots$ & 0 & 0 \\
\hline$\vdots$ & $\vdots$ & $\vdots$ & $\ddots$ & $\vdots$ & $\vdots$ \\
\hline$N_{T}$ & 0 & 0 & $\ldots$ & $\sqrt{P_{-} s_{N_{T}}}$ & 0 \\
\hline
\end{tabular}

transmit node 1 are denoted by $\tilde{s}$, while the final decisions, at the destination node, are denoted by $\hat{s}$. Note that in the $N_{T}$-th symbol period, only cooperating node 1 transmits at full power. The symbol transmitted by node 1 in the $i$-th symbol period is its decision on the symbol transmitted by node $i$ in the $i-1^{t} h$ symbol period, $i=2, \ldots, N_{T}$. For simplicity, in our strategy node $i$ transmits its symbol $s_{i}$ in the $i-1^{t} h$ cooperative symbol period, for $i=2, \ldots, N_{T}$, but these symbols could be transmitted by their corresponding nodes in any other order.

\section{B. Maximum Likelihood Detection and Its Approximate Ver- sion}

The received symbol at the destination node in the $i$-th time slot is given by

$y_{i}=\left\{\begin{aligned} \sqrt{P_{+}} s_{1} h_{1,0}+\sqrt{P_{-}} s_{2} h_{2,0}+\eta_{1}, & i=1, \\ \sqrt{P_{+}} \tilde{s}_{i} h_{1,0}+\sqrt{P_{-}} s_{i+1} h_{i+1,0}+\eta_{i}, & i=2, \ldots, N_{T}-1, \\ \sqrt{P} \tilde{s}_{N_{T}} h_{1,0}+\eta_{N_{T}}, & i=N_{T},\end{aligned}\right.$

where $\eta_{i}$ represents the AWGN term, modeled as an independent zero mean circularly symmetric complex Gaussian random variable with variance $N_{0} / 2$ per dimension.

Let $\mathbf{s}$ denote the sequence $s_{1}, \ldots, s_{N_{T}}$. Consider similar notation for the sequences $\mathbf{y}$ and $\tilde{\mathbf{s}}$. The maximum likelihood (ML) detector (MLD) at the destination node is the one yielding the decision $\left(\hat{s}_{1}, \ldots, \hat{s}_{N_{T}}\right)$ given by

$$
\begin{aligned}
& \left(\hat{s}_{1}, \ldots, \hat{s}_{N_{T}}\right)=\arg \max _{\mathbf{s}} p(\mathbf{y} \mid \mathbf{s}) \\
& =\arg \max _{\mathbf{s}} \sum_{\tilde{\mathbf{s}}} p(\mathbf{y}, \tilde{\mathbf{s}} \mid \mathbf{s}) \\
& =\arg \max _{\mathbf{s}} \sum_{\tilde{\mathbf{s}}} p(\mathbf{y} \mid \tilde{\mathbf{s}}, \mathbf{s}) p(\tilde{\mathbf{s}} \mid \mathbf{s}) \\
& =\arg \max _{\mathbf{s}} \sum_{\tilde{\mathbf{s}}} \exp \left(-\left|y_{1}-\sqrt{P_{+}} s_{1} h_{1,0}-\sqrt{P_{-}} s_{2} h_{2,0}\right|^{2}\right) \\
& \left.\quad \exp -\sum_{i=2}^{N_{T}-1}\left|y_{i}-\sqrt{P_{+}} \tilde{s}_{i} h_{1,0}-\sqrt{P_{-}} s_{i+1} h_{i+1,0}\right|^{2}\right) \\
& \quad \operatorname{exk}\left(-\left|y_{N_{T}}-\sqrt{P} \tilde{s}_{N_{T}} h_{1,0}\right|^{2}\right) \exp (\ln (p(\tilde{\mathbf{s}} \mid \mathbf{s})))
\end{aligned}
$$

By applying the max-log property, namely,

$$
\log \sum_{i} e^{a_{i}} \approx \max _{i}\left\{a_{i}\right\}
$$


we can approximate (1) as

$$
\begin{aligned}
&\left(\hat{s}_{1}, \ldots, \hat{s}_{N_{T}}\right) \approx \\
& \arg ^{*} \max _{\mathbf{s}, \tilde{\mathbf{s}}}-\left|y_{1}-\sqrt{P_{+}} s_{1} h_{1,0}-\sqrt{P_{-}} s_{2} h_{2,0}\right|^{2} \\
& \quad-\sum_{i=2}^{N_{T}-1}\left|y_{i}-\sqrt{P_{+}} \tilde{s}_{i} h_{1,0}-\sqrt{P_{-}} s_{i+1} h_{i+1,0}\right|^{2} \\
& \quad-\left|y_{N_{T}}-\sqrt{P} \tilde{s}_{N_{T}} h_{1,0}\right|^{2}+\ln (p(\tilde{\mathbf{s}} \mid \mathbf{s}))
\end{aligned}
$$

where arg* max is the standard logical function arg max except that it extracts only the values of $s_{1}, \ldots, s_{N_{T}}$ of the argument for which the given expression attains its maximum value.

We should now observe that since, in our scenario, the interuser channel is considered to be much more reliable than the direct channel (i.e., for $\tilde{\mathbf{s}} \neq \mathbf{s}, \ln (p(\tilde{\mathbf{s}} \mid \mathbf{s}))$ is a relatively large, negative quantity), it is very likely that the pair $(\tilde{\mathbf{s}}, \mathbf{s})=\left(\mathbf{s}^{\prime}, \mathbf{s}^{\prime}\right)$ will maximize (2) for some $\mathbf{s}^{\prime}$. So, the ML decision can be further approximated as

$$
\begin{aligned}
& \left(\hat{s}_{1}, \ldots, \hat{s}_{N_{T}}\right) \approx \\
& \underset{s_{1}, \ldots, s_{N_{T}}, \tilde{s}_{2}=s_{2}, \ldots, \tilde{s}_{N_{T}}=s_{N_{T}}}{\arg { }^{*}} \min \\
& \quad+\sum_{i=2}^{N_{T}-1}\left|y_{1}-\sqrt{P_{+}} s_{1} h_{1,0}-\sqrt{P_{+}} s_{2} h_{i} h_{1,0}-\sqrt{P_{-}} s_{i+1} h_{i+1,0}\right|^{2} \\
& \quad+\left|y_{N_{T}}-\sqrt{P} \tilde{s}_{N_{T}} h_{1,0}\right|^{2} .
\end{aligned}
$$

We refer to this detector as "approximate" ML detector (AMLD).

\section{Suboptimal Linear Detection}

Assuming that $P_{+} \gg P_{-}$, the AMLD can be simplified by removing the third term inside the absolute value

$$
\left|y_{i}-\sqrt{P_{+}} \tilde{s}_{i} h_{1,0}-\sqrt{P_{-}} s_{i+1} h_{i+1,0}\right|^{2},
$$

resulting in the approximation

$$
\begin{aligned}
\left|y_{i}-\sqrt{P_{+}} \tilde{s}_{i} h_{1,0}-\sqrt{P_{-}} s_{i+1} h_{i+1,0}\right|^{2} \approx\left|y_{i}\right|^{2} \\
+P_{+}\left|\tilde{s}_{i}\right|^{2}\left|h_{1,0}\right|^{2}-2 \sqrt{P_{+}} \mathfrak{R}\left\{y_{i} \tilde{s}_{i}^{*} h_{1,0}^{*}\right\},
\end{aligned}
$$

where $i=2, \ldots, N_{T}-1$. Note that since the first and the second terms of (3) become independent of $s_{i+1}$ when $P_{+} \gg$ $P_{-}$, the detection of the $N_{T}$ symbols can be performed in parallel. For symbols $s_{N_{T}-i}$, where $i=1, \ldots, N_{T}-1$, the detection rule becomes

$$
\hat{s}_{N_{T}-i}=\arg \min _{\tilde{s}_{N_{T}-i}}\left|y_{N_{T}-i}-\sqrt{P_{+}} \tilde{s}_{N_{T}-i} h_{1,0}\right|^{2},
$$

which corresponds to the ML detection of a selection combining scheme wherein the symbol $s_{N_{T}-i}$ is transmitted at almost full power.

Similarly, the detection of symbol $s_{N_{T}}$ corresponds to the ML detection of a selection combining scheme wherein the symbol $s_{N_{T}}$ is transmitted at full power $P$. Thus, the cooperative diversity order of the proposed scheme is similar to that of an $N_{T}$-level selection combining diversity scheme, i.e., a cooperative diversity order of $N_{T}$ is achieved.

If we further assume that all signals transmitted by the $N_{T}$ transmit nodes have the same energy, which is the case if a PSK signal constellation is adopted, then the proposed detector becomes linear and reduces to

$$
\hat{s}_{i}=\arg \max _{s_{i}} \Re\left\{y_{i} s_{i}^{*} h_{1,0}^{*}\right\},
$$

where $i=1, \ldots, N_{T}$. We adopt the BPSK modulation for the remainder of this paper, which allows us to refer to the proposed detector as the linear detector (LD).

As we will see later on in this paper, the LD performance is similar to that of the MLD when the number of cooperating nodes is small. For an improved error performance with a reasonably low complexity, we present next an alternative receiver whose performance is similar to the ML performance even for a larger number of cooperating nodes.

\section{Reduced Complexity Interference Cancelation Detector}

The alternative detector we propose first performs detection of $s_{N_{T}}$ based on the minimization

$$
\begin{aligned}
\hat{s}_{N_{T}}= & \arg \min _{\tilde{s}_{N_{T}}}\left|y_{N_{T}}-\sqrt{P} \tilde{s}_{N_{T}} h_{1,0}\right|^{2} \\
= & \arg \min _{\tilde{s}_{N_{T}}}\left|y_{N_{T}}\right|^{2}+P\left|\tilde{s}_{N_{T}}\right|^{2}\left|h_{1,0}\right|^{2} \\
& -2 \sqrt{P} \Re\left\{y_{N_{T}} \tilde{s}_{N_{T}}^{*} h_{1,0}^{*}\right\},
\end{aligned}
$$

which corresponds to performing minimization of the third term alone in (3). Then $s_{N_{T}-i}$ could be detected by removing from $y_{N_{T}-i}$ the interference induced by $s_{N_{T}-i+1}$, $i=1, \ldots, N_{T}-1$, for which the detected symbol $\hat{s}_{N_{T}-i+1}$ in the previous step could be used. The decision on $s_{N_{T}-i}$ would then be given by

$$
\begin{aligned}
& \hat{s}_{N_{T}-i}=\arg \min _{\tilde{s}_{N_{T}-i}} \\
& \left|y_{N_{T}-i}-\sqrt{P_{+}} \tilde{s}_{N_{T}-i} h_{1,0}-\sqrt{P_{-}} \hat{s}_{N_{T}-i+1} h_{N_{T}-i+1,0}\right|^{2}
\end{aligned}
$$

It should be remarked that this is just the detection based on interference cancelation for the multiple access channel, which is well-known [19]. The novelty, which will appear in the performance analysis of Section IV, comes from the fact that the channels are neither Gaussian nor standard fading (Rayleigh or Rice) channels, but rather channels whose statistics are based upon ordered random variables. We refer to this detector as the interference cancelation detector (ICD).

\section{Performance AnAlysis}

In this section, we derive an upper bound on the average bit error rate (BER) expression for the cooperative diversity scheme described in Section III. In particular, we focus our analysis on the case of the ICD. Assume for simplicity that the transmit nodes use BPSK modulation.

Before we begin the derivation of the BER expression, we present the notation for the probabilities that will be considered in this section. The probability of symbol detection error at node 1 will be denoted as $P^{1}$. The probability of symbol 
detection error at destination node (node 0) assuming that the correct symbol is transmitted with power $P$ by node 1 will be denoted as $P^{0}$. According to Table I, the only symbol transmitted by node 1 with power $P$ is the symbol $\tilde{s}_{N_{T}}$, and it is the correct symbol if it has the same value as symbol $s_{N_{T}}$. The probability of symbol detection error at destination node (node 0 ) assuming that the correct symbol is transmitted with power $P_{+}$by node 1 while another symbol, an estimation of which is assumed available for interference removal at the destination node, is simultaneously transmitted with power $P_{-}$by some other cooperative node will be denoted as $P^{0, i}$, where the superscript $i$ stands for interference. According to Table I, the symbols transmitted by node 1 with power $P^{+}$ and subject to interference are the symbols $s_{1}$ and $\tilde{s}_{k}$, for $k=2, \ldots, N_{T}-1$, where $\tilde{s}_{k}$ is the correct symbol if it has the same value of symbol $s_{k}$. The probability of detection error for a specific symbol $s$ will be denoted as $P_{s}$. Instantaneous probabilities will be denoted either as $P_{\mid h}$ or $P_{s \mid h}$.

We begin our analysis by presenting the average probability of symbol detection error at node 1 . For BPSK modulation and assuming the Rician fading model for the inter-user channels, the average probability of symbol detection error at node 1 is given by [20]:

$$
\begin{aligned}
P^{1}\left(\gamma_{1}\right)= & \frac{1}{\pi} \int_{0}^{\frac{\pi}{2}} \frac{(1+K) \sin ^{2}(\varphi)}{(1+K) \sin ^{2}(\varphi)+\sin ^{2}\left(\frac{\pi}{2}\right) \gamma_{1}} \\
& \left.\quad \operatorname{kxp} \frac{K \sin ^{2}\left(\frac{\pi}{2}\right) \gamma_{1}}{(1+K) \sin ^{2}(\varphi)+\sin ^{2}\left(\frac{\pi}{2}\right) \gamma_{1}}\right) d \varphi
\end{aligned}
$$

where $K$ is the Rician parameter, $\gamma_{1}=P_{-}$ISNR, and ISNR is the average signal-to-noise ratio (SNR) of the inter-user channel.

The average probability of symbol detection error at destination node assuming that the correct symbol is transmitted with power $P$ by node 1 is that of a $N_{T}$-level selection diversity combining scheme [21]:

$$
\begin{aligned}
P^{0}\left(\gamma_{0}\right)= & \int_{0}^{\infty} Q\left(\sqrt{2 \gamma_{b}}\right) p\left(\gamma_{b}\right) d \gamma_{b} \\
= & N_{T} \sum_{k=0}^{N_{T}-1} \frac{(-1)^{k}\left(\begin{array}{c}
N_{T}-1 \\
k
\end{array}\right)}{2(k+1)} \\
& \left(1-\sqrt{\frac{\gamma_{0}}{\gamma_{0}+k+1}}\right),
\end{aligned}
$$

where $p\left(\gamma_{b}\right)$ is the probability density function of the random variable $\gamma_{b}=\gamma_{0} P\left|h_{1,0}\right|^{2}, \gamma_{0}=1 / N_{0}$ is the average SNR of the forward channel, $Q\left(\sqrt{2 \gamma_{b}}\right)$ is the instantaneous BER based on a single channel realization, and $\left|h_{1,0}\right|^{2}$ is the $N_{T}$-th order statistics [22] of the channel coefficients squared norms, i.e., it is the random variable representing the largest of $N_{T}$ chi-squared random variables with 2 degrees of freedom and unit expected value.

The instantaneous BER for the symbol $s_{N_{T}}$ is given by

$$
P_{s_{N_{T}} \mid h}=P_{\mid h}^{0}\left(\gamma_{b}\right)\left(1-P_{\mid h}^{1}\right)+P_{\mid h}^{1}\left(1-P_{\mid h}^{0}\left(\gamma_{b}\right)\right),
$$

where

$$
P_{\mid h}^{0}\left(\gamma_{b}\right)=Q\left(\sqrt{2 \gamma_{b}}\right)
$$

is the instantaneous probability that the symbol $s_{N_{T}}$ is detected erroneously by the destination node assuming that it was detected correctly by the best node, and $P_{\mid h}^{1}$ is the instantaneous probability that the symbol sent by a cooperative node is detected erroneously by the best node. $P_{\mid h}^{0}\left(\gamma_{b}\right)$ depends on $h_{1,0}$ (which is a function of all $h_{i, 0}$ 's), while $P_{\mid h}^{1}$ depends solely on the $h_{i, 1}$ 's. Since by the assumption, $h_{i, 0}$ and $h_{i, 1}$ are statistically independent, the average of (10) is given by

$$
\begin{aligned}
P_{s_{N_{T}}}\left(\gamma_{0}, \gamma_{1}\right)= & P^{0}\left(\gamma_{0}\right)\left(1-P^{1}\left(\gamma_{1}\right)\right) \\
& +P^{1}\left(\gamma_{1}\right)\left(1-P^{0}\left(\gamma_{0}\right)\right) .
\end{aligned}
$$

We now present the probability of symbol detection error at

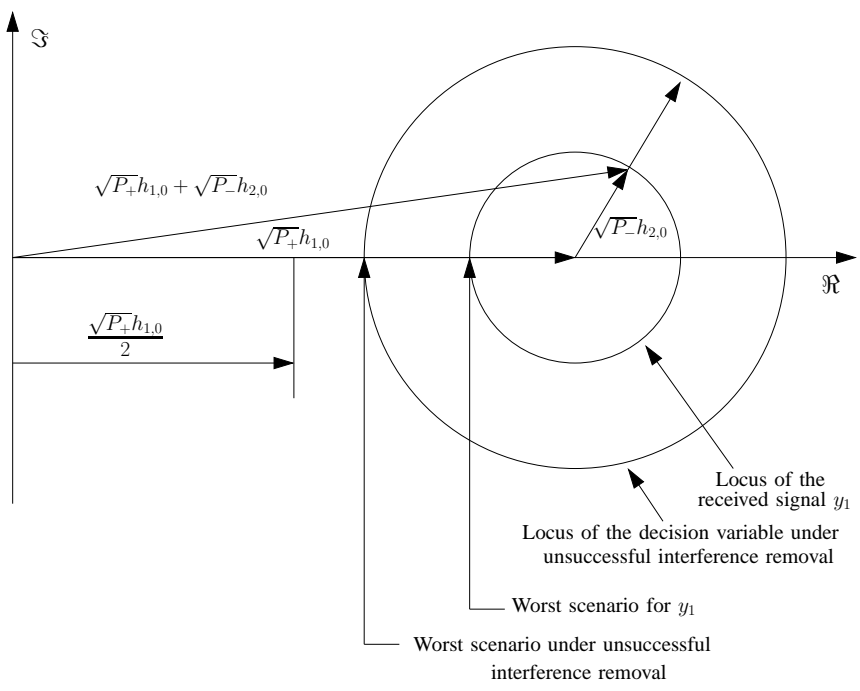

Fig. 2. $\quad \hat{s}_{i}$ under the assumption $P_{+} \geq 16 P_{-}$for BPSK. The phasors shown are for the case where $s_{i}$ and $s_{i+1}$ are equal, $i=1, \ldots, N_{T}-1$.

the destination node assuming that the correct symbol $\left(\tilde{s}_{k}=\right.$ $\left.s_{k}, k=2, \ldots, N_{T}-1\right)$ is transmitted with power $P_{+}$by node 1 while another symbol $s_{k+1}$ is simultaneously transmitted with power $P_{-}$by another cooperative node. It is also assumed that an estimate of this symbol is available at this time at the destination node for interference removal. This instantaneous BER for the symbol $s_{k}$, for $k=N_{T}-1, \ldots, 1$, is given by

$$
\begin{aligned}
P_{s_{k} \mid h}^{0, i}= & \left(1-P_{s_{k+1} \mid h}^{0, i}\left(\gamma_{b}^{+}\right)\right) Q\left(\sqrt{2 \gamma_{b}^{+}}\right) \\
& +\frac{P_{s_{k+1} \mid h}^{0, i}\left(\gamma_{b}^{+}\right)}{2} \sum_{j=0}^{1} Q\left(\sqrt{\frac{2\left|\Pi_{h_{1,0}}\left(\Delta_{j}\right)\right|^{2}}{N_{0}}}\right),
\end{aligned}
$$

where $P_{s_{k+1} \mid h}^{0, i}\left(\gamma_{b}^{+}\right)$is the probability that the interference is not properly removed, $\gamma_{b}^{+}=\frac{P^{+}}{P} \gamma_{b}, \Delta_{j}=\sqrt{P_{+}} h_{1,0}+$ $2(-1)^{j} \sqrt{P_{-}} h_{k, 0}$, and $\Pi_{h_{1,0}}\left(\Delta_{j}\right)$ denotes the projection of $\Delta_{j}$ on the real axis, as illustrated in Figure 2.

In the second term of (13), we consider the fact that, for BPSK modulation, if the interference is not properly removed the remaining interference is twice as high. The index $j$ of $\Delta_{j}$ indicates whether the symbols $s_{i}$ and $s_{i+1}$ have the same $(j=0)$ or opposite $(j=1)$ values.

Next, a series of inequalities will be presented aiming at deriving an upper bound on the sum of $Q$-functions in (13). 
For either $j=0$ or $j=1$, we can easily show that

$$
\left|\Pi_{h_{1,0}}\left(\Delta_{j}\right)\right|^{2} \geq\left(\sqrt{P_{+}}\left|h_{1,0}\right|-2 \sqrt{P_{-}}\left|h_{k, 0}\right|\right)^{2}
$$

where equality holds if and only if $h_{1,0}\left|h_{k, 0}\right|=-h_{k, 0}\left|h_{1,0}\right|$. The right-hand side of the inequality above corresponds to the worst scenario under unsuccessful interference removal, as illustrated in Figure 2. If we assume further that $P_{+} \geq 16 P_{-}$ (or $P_{+} \geq 16 \frac{\left|h_{k, 0}\right|^{2}}{\left|h_{1,0}\right|^{2}} P_{-}$) (see Figure 2), then we have the last inequality of the series, namely,

$$
\left(\sqrt{P_{+}}\left|h_{1,0}\right|-2 \sqrt{P_{-}}\left|h_{k, 0}\right|\right)^{2} \geq \frac{P_{+}}{4}\left|h_{1,0}\right|^{2} .
$$

This means that (13) can be upper bounded as

$$
\begin{aligned}
P_{s_{k} \mid h}^{0, i} \leq & \left(1-P_{s_{k+1} \mid h}^{0, i}\left(\gamma_{b}^{+}\right)\right) Q\left(\sqrt{2 \gamma_{b}^{+}}\right) \\
& +P_{s_{k+1} \mid h}^{0, i}\left(\gamma_{b}^{+}\right) Q\left(\sqrt{\frac{\gamma_{b}^{+}}{2}}\right) .
\end{aligned}
$$

The probability that the interference is not properly removed $P_{s_{k+1} \mid h}^{0, i}\left(\gamma_{b}^{+}\right)$is the probability that the symbol $s_{k+1}$ is detected erroneously by the destination node in the previous step. So, the instantaneous BER can be obtained recursively. However, this creates a series of dependencies that will make it difficult to evaluate the average BER. We can then use the argument that since $Q\left(\sqrt{2 \gamma_{b}^{+}}\right)$is smaller than $Q\left(\sqrt{\gamma_{b}^{+} / 2}\right)$, then (14) is an increasing function of $P_{s_{k+1} \mid h}^{0, i}$. Therefore, (14) can be further upper-bounded if we substitute the probability $P_{s_{k+1} \mid h}^{0, i}$ in (14) by an upper bound. While this upper bound can be loose to express the probability that the symbol $s_{k+1}$ is detected erroneously by the destination node, it is good enough to obtain a tight upper bound on $P_{s_{k} \mid h}^{0, i}$ when used in (14). The probability $P_{s_{k+1} \mid h}^{0, i}\left(\gamma_{b}^{+}\right)$which appears in (14) can be upper bounded as

$$
P_{s_{k+1} \mid h}^{0, i}\left(\gamma_{b}^{+}\right) \leq\left(N_{T}-k\right) Q\left(\sqrt{2 \gamma_{b}^{+}}\right) .
$$

From the above, the instantaneous BER for the symbol $s_{k}$, $k=1, \ldots, N_{T}-1$, assuming that the correct symbol $\left(\tilde{s}_{k}=s_{k}\right)$ is transmitted with power $P_{+}$by node 1 while another symbol is simultaneously transmitted with power $P_{-}$by some other cooperative node can be upper bounded as

$$
\begin{aligned}
P_{s_{k} \mid h}^{0, i} \leq & \left(1-\left(N_{T}-k\right) Q\left(\sqrt{2 \gamma_{b}^{+}}\right)\right) Q\left(\sqrt{2 \gamma_{b}^{+}}\right) \\
& +\left(N_{T}-k\right) Q\left(\sqrt{2 \gamma_{b}^{+}}\right) Q\left(\sqrt{\frac{\gamma_{b}^{+}}{2}}\right) .
\end{aligned}
$$

The corresponding upper bound for the average BER can be obtained by taking the expectation of (15) resulting in

$$
\begin{aligned}
P_{s_{k}}^{0, i} \leq & E\left\{Q\left(\sqrt{2 \gamma_{b}^{+}}\right)\right\}-\left(N_{T}-k\right) E\left\{Q\left(\sqrt{2 \gamma_{b}^{+}}\right)^{2}\right\} \\
+ & \left(N_{T}-k\right) E\left\{Q\left(\sqrt{\frac{\gamma_{b}^{+}}{2}}\right) Q\left(\sqrt{2 \gamma_{b}^{+}}\right)\right\} .
\end{aligned}
$$

The final result is left as a function of $P^{0}\left(\gamma_{0}\right)$ in (9) and of $\Omega(\alpha, \beta)$, defined as

$$
\Omega(\alpha, \beta)=E\left\{Q\left(\sqrt{\alpha\left|h_{1,0}\right|^{2}}\right) Q\left(\sqrt{\beta\left|h_{1,0}\right|^{2}}\right)\right\},
$$

for which a closed-form expression is derived in the Appendix. Then,

$$
\begin{aligned}
P_{s_{k}}^{0, i}\left(\gamma_{0}\right) \leq & P^{0}\left(\gamma_{0} P_{+} / P\right)+\left(N_{T}-k\right) \\
& {\left[\Omega\left(\gamma_{0} P_{+} / 2,2 \gamma_{0} P_{+}\right)-\Omega\left(2 \gamma_{0} P_{+}, 2 \gamma_{0} P_{+}\right)\right] . }
\end{aligned}
$$

Now, considering the received signal in the interval $k(k=$ $\left.2, \ldots, N_{T}-1\right)$

$$
y_{k}=\sqrt{P_{+}} \tilde{s}_{k} h_{1,0}+\sqrt{P_{-}} s_{k+1} h_{k+1,0}+\eta_{k},
$$

where $\tilde{s}_{k}$ is the decision on $s_{k}$ taken by node 1 in the instant $k-1$, the probability that $\tilde{s}_{k}$ is not equal to $s_{k}$ is $P^{1}$. The instantaneous probability $P_{s_{k} \mid h}$ that node 0 makes an incorrect decision on $s_{k}$ is given by:

$$
P_{s_{k} \mid h}=P_{s_{k} \mid h}^{0, i}\left(1-P_{\mid h}^{1}\right)+P_{\mid h}^{1}\left(1-P_{s_{k} \mid h}^{0, i}\right),
$$

where $k=2, \ldots, N_{T}-1$, and $P_{s_{k} \mid h}^{0, i}$ is given by (15). We should note that the bound on $P_{s_{k} \mid h}^{0, i}$ in (15) depends only on $h_{1,0}$ (and $h_{2,0}, h_{3,0}, \ldots, h_{N_{T}, 0}$ ), while $P_{\mid h}^{1}$ depends only on $h_{k, 1}, k=2, \ldots, N_{T}-1$. Therefore, the average probability of error for the symbol $s_{k}$ is given by

$$
\begin{array}{r}
P_{s_{k}}\left(\gamma_{0}, \gamma_{1}\right)=P_{s_{k}}^{0, i}\left(\gamma_{0}\right)\left(1-P^{1}\left(\gamma_{1}\right)\right)+ \\
P^{1}\left(\gamma_{1}\right)\left(1-P_{s_{k}}^{0, i}\left(\gamma_{0}\right)\right),
\end{array}
$$

where $P_{s_{k}}^{0, i}\left(\gamma_{0}\right)$ is the average BER in (17). Finally, the probability of detection error of the symbol $s_{1}$ can only be lower than (20), because node 1 owns the symbol $s_{1}$. So, the bound in (20) can be extended to $k=1$.

The final result of this section, namely, the average BER for BPSK corresponding to the ICD for the new cooperative scheme, can be upper bounded by the arithmetic mean of (12) and (20).

\section{Simulation Results}

In this section, we present several simulation results to assess the error performance of the communication system with cooperation proposed in this paper (presented in Section III). In all the simulations, we present the BER versus SNR $\left(\gamma_{0}\right)$ for BPSK modulation, assuming Rayleigh flat fading for the forward channels.

First, in Figure 3, we compare the proposed scheme for $b_{f}=1, N_{T}=2$, with the Alamouti cooperative diversity scheme [23] (full-duplex channel, without feedback). In this figure, we assume ideal inter-user channels. In order to show the cooperative diversity gain, the BER curve for the scheme without cooperation (each node transmits its own symbol at separate symbol intervals) is also shown in Figure 3. Results are shown for both the LD and the MLD. The theoretical upper bound is also shown to give an idea of its tightness. The power allocation adopted in this simulation is $P_{+}=0.95$, where the total power was set to $P=1$. It can be seen that the linear detector has an excellent performance. We also observe 


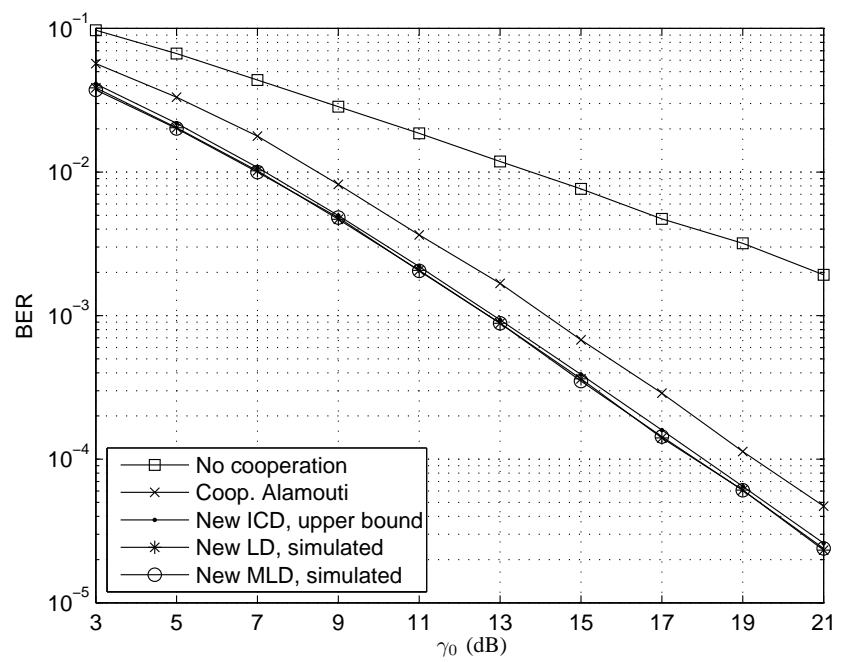

Fig. 3. BER versus SNR for the new cooperative scheme and the Alamouti cooperative diversity scheme [23] (full-duplex channel, without feedback), for $N_{T}=2$, ideal inter-user channels, and $P_{+}=0.95$.

that by using only one feedback bit in this case the proposed scheme presents an SNR gain over the cooperative scheme with Alamouti code of about $1.5 \mathrm{~dB}$.

In Figure 4, the cooperative diversity scheme with the G4 code [5] (full-duplex channel, without feedback) and the new cooperative diversity scheme for $b_{f}=2$ feedback bits are considered, assuming that the inter-user channels are subject to Rician fading, with $K=20 \mathrm{~dB}$ and ISNR $=25 \mathrm{~dB}$. The results are shown for the ICD receiver. The theoretical upper bound is shown as well. The power allocation adopted in this simulation is $P_{+}=0.95$, where the total power is $P=1$. We should note that the proposed cooperative diversity scheme, with $b_{f}=2$ feedback bits, shows an SNR gain over the cooperative diversity scheme with code $\mathrm{G} 4$ of about $2.5 \mathrm{~dB}$.

Figure 5 gives the theoretical error performance curves for the ICD receiver, for different values of $N_{T}$. We can observe that the theoretical upper bound curves for the BER expression are very close to the simulation performance curves obtained when we consider the ICD receiver, even when $N_{T}$ is increased.

Figure 6 shows the error results for the ICD and LD receivers, for different values of $N_{T}$. We can observe that as $N_{T}$ is increased, the linear receiver shows some performance loss. However, it is still an attractive choice due to the simplicity of detection.

We remark that, in the case of ideal inter-user channel, the power allocation $P_{+}$could be arbitrarily high, which would improve even further the error performance of the new cooperative scheme. However, for more realistic scenarios, the power $P_{-}$cannot be too low since the decision errors at the partner node would compromise the overall system's performance. For this situation, we have simulated two scenarios. In Figures 7 and 8 , we considered $N_{T}=2$ transmit nodes and Rician inter-user fading channels with an ISNR $=\mathrm{SNR}+5 \mathrm{~dB}$ and $\mathrm{ISNR}=\mathrm{SNR}+10 \mathrm{~dB}$, respectively. For the first and second

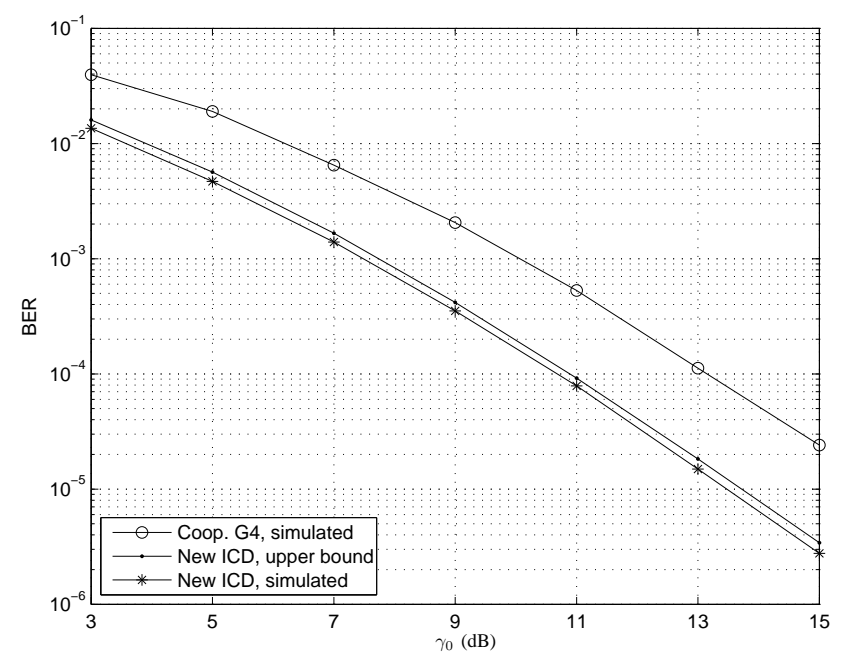

Fig. 4. BER versus SNR for the new cooperative scheme and the cooperative diversity scheme with the G4 code [5] (full-duplex channel, without feedback), for $N_{T}=4$, ISNR $=25 \mathrm{~dB}, K=20 \mathrm{~dB}$, and $P_{+}=0.95$.

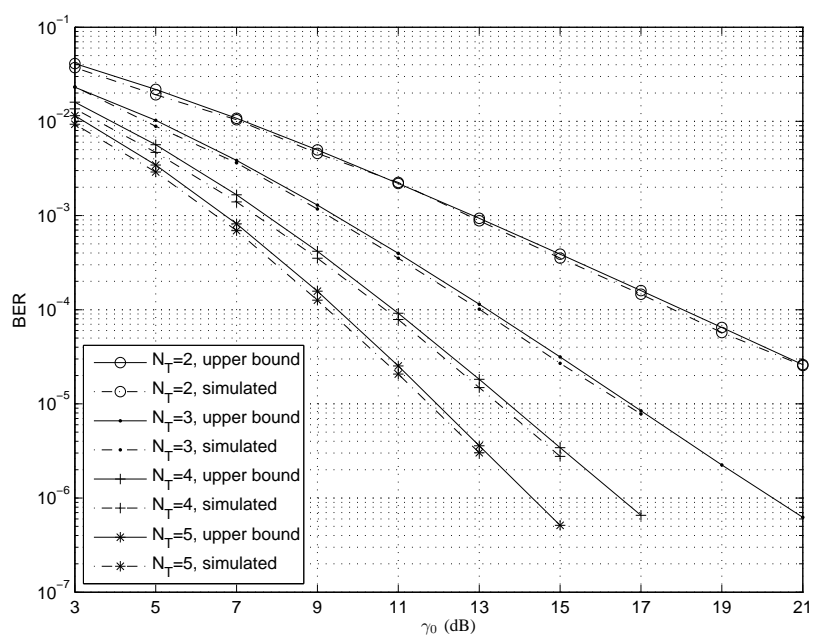

Fig. 5. BER versus SNR for the new cooperative scheme with the ICD, for $N_{T}=2,3,4$ and $5 . K=20 \mathrm{~dB}$, ISNR $=25 \mathrm{~dB}$, and $P_{+}=0.95$. Dashed lines are for the simulated BER and the solid lines are for the theoretical upper bounds.

scenarios, respectively, the optimal $P_{+}$was found in the range from 0.65 to 0.95 and from 0.85 to 0.95 , with increasing value as the SNR is increased. The optimal power allocation as a function of the inter-user channel statistics and average SNR at destination node was obtained by making use of the theoretical upper bound on the BER derived earlier.

\section{CONCLUSION AND FINAL COMMENTS}

In this paper, we have proposed a simple cooperative diversity scheme for a communication system consisting of $N_{T} \geq 2$ cooperating nodes that receive $\left\lceil\log _{2}\left(N_{T}\right)\right\rceil$ CSI bits from the destination node. These feedback bits indicate which cooperating node has the strongest channel, and this information is 


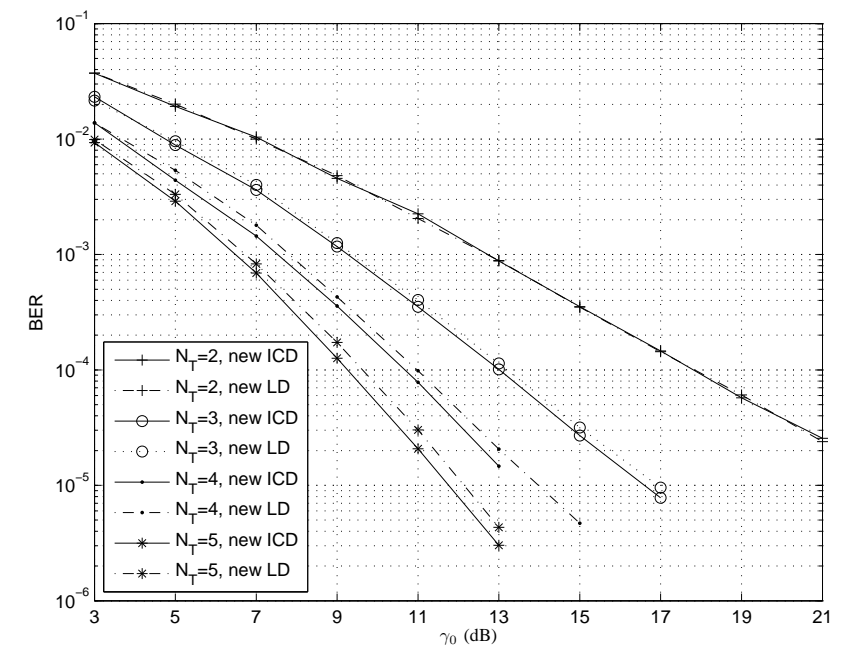

Fig. 6. BER versus SNR for the proposed cooperative scheme with the ICD (solid lines) and the LD (dashed lines), for $N_{T}=2,3,4$ and $5 . K=20 \mathrm{~dB}$, ISNR $=25 \mathrm{~dB}$, and $P_{+}=0.95$. All curves were obtained from simulation.

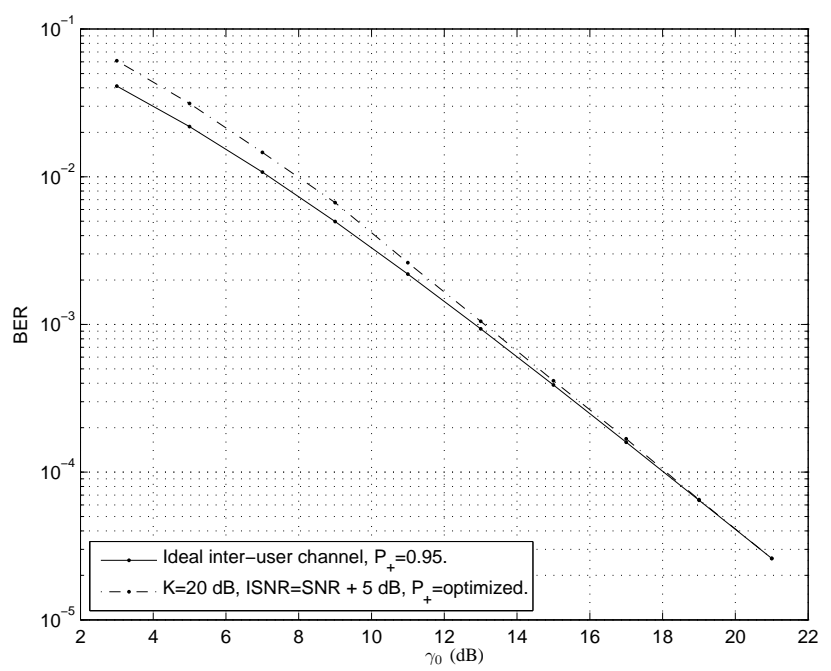

Fig. 7. BER versus SNR for the new cooperative scheme with the ICD for $N_{T}=2$. Solid line shows the performance for the ideal inter-user channel case with $P_{+}=0.95$ and the dashed line shows the performance for the non-ideal inter-user channel case, where $K=20 \mathrm{~dB}$, ISNR $=\mathrm{SNR}+5 \mathrm{~dB}$, and $P_{+}$is optimized offline.

used appropriately to obtain cooperative diversity and SNR gains. A simple interference cancelation detector and a linear detector are proposed and their performances are shown to be very close to the maximum likelihood error performance. An upper bound on the average error probability for binary phaseshift keying in flat Rayleigh channels under the assumption of Rician inter-user channels is derived. Comparisons in terms of BER versus SNR between the ICD receiver and the LD receiver are also made. Employing the simulation results and the performance analysis, it is shown that the maximum diversity order (equal to $N_{T}$ ) is achieved by the proposed scheme. When

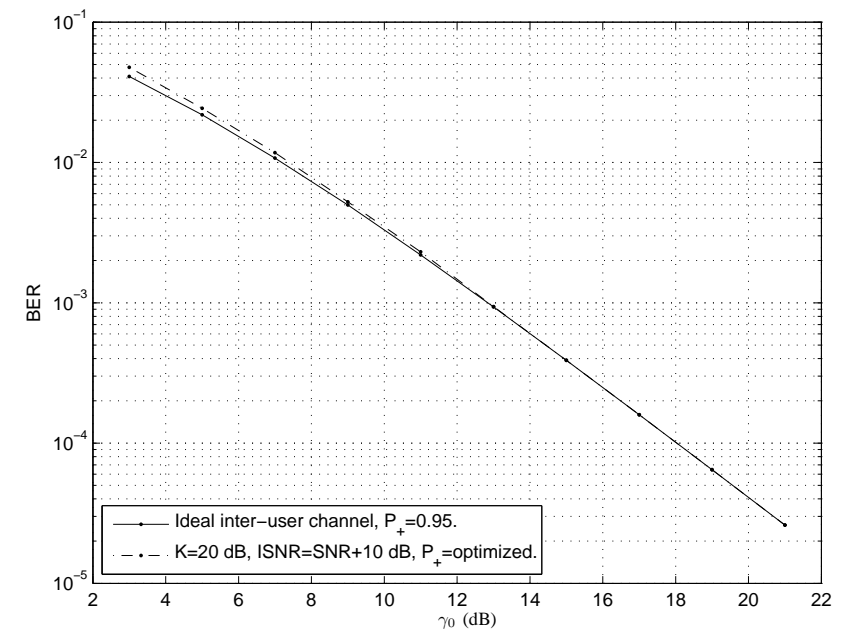

Fig. 8. BER versus SNR for the proposed cooperative scheme with the ICD for $N_{T}=2$. Solid line shows the performance for the ideal inter-user channel case with $P_{+}=0.95$ and the dashed line shows the performance for the non-ideal inter-user channel case, where $K=20 \mathrm{~dB}, \mathrm{ISNR}=\mathrm{SNR}+10$ $\mathrm{dB}$, and $P_{+}$is optimized offline.

$N_{T}=2$ and 4 , the new cooperative diversity scheme, making use of only 1 and 2 feedback bits, respectively, has an SNR gain of about $1.5 \mathrm{~dB}$ and $2.5 \mathrm{~dB}$ over the cooperative scheme without feedback that makes use of the Alamouti code and space-time code G4, respectively. Optimal power allocation as a function of inter-user channel statistics and average SNR at destination node is obtained by the use of the theoretical upper bound on the BER derived. The results presented in this paper allow us to conclude that even if the inter-user channels are not ideal, when the cooperating users are sufficiently close to each other, the performance gain is still very significant.

\section{APPENDIX}

In this appendix we derive a closed form expression for

$$
\Omega(\alpha, \beta)=E\{Q(\sqrt{\alpha X}) Q(\sqrt{\beta X})\},
$$

as a function of $N_{T}$. Consider the random variable $X=$ $\max _{k}\left|h_{k, 0}\right|^{2}$ and let $\alpha$ and $\beta$ be positive real numbers. The expectation above is with respect to $X$, whose PDF is [9], [24]:

$$
p_{X}(x)=N_{T}\left(1-e^{-x}\right)^{N_{T}-1} e^{-x}, \quad x \geq 0 .
$$

The moment generating function of $X$, denoted as $\Psi_{X}(v)$, can be defined for $v \geq 0$ as

$$
\Psi_{X}(v):=E\{\exp (-v X)\}=\int_{0}^{\infty} e^{-v x} p_{X}(x) d x,
$$

which evaluates to

$$
\Psi_{X}(v)=N_{T} \sum_{k=0}^{N_{T}-1} \frac{(-1)^{k}\left(\begin{array}{c}
N_{T}-1 \\
k
\end{array}\right)}{k+1+v} .
$$


We now recall Craig's formula [25] for the Gaussian $Q$ function:

$$
Q(x)=\frac{1}{\pi} \int_{0}^{\pi / 2} \exp \left(-\frac{x^{2}}{2 \sin ^{2} \theta}\right) d \theta .
$$

Also, it has been shown [21, eq. (4.8)] that

$$
\begin{array}{r}
Q\left(x_{1}\right) Q\left(y_{1}\right)=\frac{1}{2 \pi} \int_{0}^{\tan ^{-1} y_{1} / x_{1}} \exp \left(-\frac{y_{1}^{2}}{2 \sin ^{2} \theta}\right) d \theta \\
+\frac{1}{2 \pi} \int_{0}^{\pi / 2-\tan ^{-1} y_{1} / x_{1}} \exp \left(-\frac{x_{1}^{2}}{2 \sin ^{2} \theta}\right) d \theta .
\end{array}
$$

By substituting $x_{1}=\sqrt{\alpha X}$ and $y_{1}=\sqrt{\beta X}$ in (22), we have

$$
\begin{gathered}
Q(\sqrt{\alpha X}) Q(\sqrt{\beta X})=\frac{1}{2 \pi} \int_{0}^{\kappa_{1}} \exp \left(-\frac{\beta X}{2 \sin ^{2} \theta}\right) d \theta \\
+\frac{1}{2 \pi} \int_{0}^{\kappa_{2}} \exp \left(-\frac{\alpha X}{2 \sin ^{2} \theta}\right) d \theta
\end{gathered}
$$

where $\kappa_{1}=\tan ^{-1} \sqrt{\beta / \alpha}$ and $\kappa_{2}=\frac{\pi}{2}-\tan ^{-1} \sqrt{\beta / \alpha}$.

Taking the expectation of (23), and using (21), we obtain

$$
\Omega(\alpha, \beta)=\Omega_{1}(\alpha, \beta)+\Omega_{2}(\alpha, \beta),
$$

where

$$
\Omega_{1}(\alpha, \beta)=\frac{1}{2 \pi} \int_{0}^{\kappa_{1}} \Psi_{X}\left(\frac{\beta}{2 \sin ^{2} \theta}\right) d \theta
$$

and

$$
\Omega_{2}(\alpha, \beta)=\frac{1}{2 \pi} \int_{0}^{\kappa_{2}} \Psi_{X}\left(\frac{\alpha}{2 \sin ^{2} \theta}\right) d \theta .
$$

From (21) and the above integrals, it can be seen that we will need to solve an integral of the form

$$
\int \frac{V(k)}{C+\frac{\beta}{2 \sin ^{2} \theta}} d \theta=\frac{V(k)}{2 C} \int \frac{4 C \sin ^{2} \theta+2 \beta-2 \beta}{\beta+2 C \sin ^{2} \theta} d \theta
$$

which evaluates to [26]:

$$
\begin{aligned}
& \frac{V(k) \theta}{C}-\frac{V(k) \sqrt{\beta}}{C \sqrt{2 C+\beta}} \tan ^{-1}\left(\sqrt{1+\frac{2 C}{\beta}} \tan (\theta)\right), \\
& V(k)=N_{T}(-1)^{k}\left(\begin{array}{c}
N_{T}-1 \\
k
\end{array}\right)
\end{aligned}
$$

and $k=C-1$.

From (27) and (28), we can write (25) and (26) as

$$
\begin{aligned}
& \Omega_{1}(\alpha, \beta)=\frac{1}{2 \pi} \tan ^{-1}\left(\sqrt{\frac{\beta}{\alpha}}\right) \\
& -\sum_{i=0}^{N_{T}-1} \frac{V(i) \sqrt{\beta}}{C 2 \pi \sqrt{\beta+2 C}} \tan ^{-1}\left(\sqrt{\frac{\beta+2 C}{\alpha}}\right)
\end{aligned}
$$

and

$$
\begin{aligned}
& \begin{aligned}
\Omega_{2}(\alpha, \beta)= & \frac{1}{4}-\frac{1}{2 \pi} \tan ^{-1}\left(\sqrt{\frac{\beta}{\alpha}}\right) \\
& -\sum_{i=0}^{N_{T}-1} \frac{V(i) \sqrt{\alpha}}{C 2 \pi \sqrt{\alpha+2 C}} \tan ^{-1}\left(\sqrt{\frac{\alpha+2 C}{\beta}}\right),
\end{aligned} \\
& \text { where } C=1, \ldots, N_{T} .
\end{aligned}
$$

\section{ACKNOWLEDGEMENTS}

This work was supported in part by the Brazilian National Council for Scientific and Technological Development (CNPq) and in part by the Brazilian Research and Innovation Network in Digital Technologies (RENAPI).

\section{REFERENCES}

[1] A. Sendonaris, E. Erkip, and B. Aazhang, "User cooperation diversity Part I: System description,” IEEE Trans. Commun., vol. 51, no. 11, pp. 1927-1938, November 2003.

[2] — "User cooperation diversity - Part II: Implementation aspects and performance analysis," IEEE Trans. Commun., vol. 51, no. 11, pp. 19391948, November 2003.

[3] J. N. Laneman, G. W. Wornell, and D. N. C. Tse, "An efficient protocol for realizing cooperative diversity in wireless networks," in Proc. IEEE Int. Symp. Inf. Theory (ISIT), Washington, DC, June 2001, p. 294.

[4] J. N. Laneman, D. N. C. Tse, and G. W. Wornell, "Cooperative diversity in wireless networks: Efficient protocols and outage behavior,' IEEE Trans. Inf. Theory, vol. 50, no. 12, pp. 3062-3080, December 2004.

[5] V. Tarokh, H. Jafarkhani, and A. Calderbank, "Space-time block codes from orthogonal designs," IEEE Trans. Inform. Theory, vol. 45, no. 5, pp. 1456-1467, July 1999.

[6] S. M. Alamouti, "A simple transmit diversity technique for wireless communications," IEEE J. Select. Areas Commun, vol. 16, pp. 14511458, October 1998.

[7] E. G. Larsson and B. R. Vojcic, "Cooperative transmit diversity based on superposition modulation," IEEE Comm. Letters, vol. 9, no. 9, pp. 778-780, September 2005.

[8] G. Caire, G. Taricco, and E. Biglieri, "Optimum power control over fading channels," IEEE Trans. Inf. Theory, vol. 45, no. 5, pp. $31468-$ 1489, July 1998.

[9] D. Gore and A. Paulraj, "MIMO antenna subset selection with spacetime coding," IEEE Trans. Signal Processing, vol. 50, no. 10, pp. 25802588, December 2002.

[10] T. M. Duman and A. Ghrayeb, Coding for MIMO Communication Systems. New York: John Wiley \& Sons, 2007.

[11] T. E. Hunter and A. Nosratinia, "Diversity through coded cooperation," IEEE Trans. Wireless Commun., vol. 5, no. 2, pp. 283-289, February 2006.

[12] Y. Cao, B. Vojcic, and M. Souryal, "User-cooperative transmission with channel feedback in slow fading environment," in Proc. IEEE Vehicular Technology Conf. (VTC-Fall), Los Angeles, CA, September 2004, pp. 2063-2067.

[13] N. Ahmed, M. A. Khojastepour, A. Sabharwal, and B. Aazhang, "Outage minimization with limited feedback for the fading relay channel," IEEE Trans. Commun., vol. 54, no. 4, pp. 659-669, April 2006.

[14] A. Bletsas, A. Khisti, D. Reed, and A. Lippman, "A simple cooperative diversity method based on network path selection," IEEE Journal on Selected Areas of Commun. (JSAC), vol. 24, no. 3, pp. 659-672, March 2006.

[15] T. Riihonen, R. Wichman, and J. Hämäläinen, "Co-phasing full-duplex relay link with non-ideal feedback information," in Proc. IEEE Int. Symp. on Wireless Commun. Syst. (ISWCS), Reykjavik, Iceland, October 2008, pp. 263-267.

[16] O. Somekh, O. Simeone, H. V. Poor, and S. Shamai, "Cellular systems with full-duplex amplify-and-forward relaying and cooperative basestations," in Proc. IEEE Int. Symp. Inform. Theory (ISIT), Washington, DC, June 2007, pp. 16-20.

[17] A. Zaidi, S. Kotagiri, J. N. Laneman, and L. Vandendorpe, "Cooperative relaying with state available at the relay," in Proc. IEEE Inform. Theory Workshop (ITW), Porto, Portugal, May 2008, pp. 139-143.

[18] H. Ju, E. Oh, and D. Hong, "Improved efficiency of resource usage in two-hop full duplex relay systems based on resource sharing and interference cancellation," IEEE Trans. Wireless Commun., vol. 8, no. 8, pp. 3933-3938, August 2009.

[19] T. M. Cover and J. A. Thomas, Elements of Information Theory. 2nd edition: New York: Wiley, 1991.

[20] A. Goldsmith, Wireless Communications. Cambridge: Cambridge University Press, 2005.

[21] M. K. Simon and M. S. Alouini, Digital Communications over Fading Channels. New York: John Wiley \& Sons, Inc., 2000.

[22] H. A. David and H. N. Nagaraja, Order Statistics. New York: John Wiley \& Sons, 2003. 
[23] L. Tao, L. Xiang-Ming, and Y. Guang-Xin, "Performance estimating for space-time block coded cooperative communication system," in Proc. IEEE Int. Conf. on Commun., Circuits and Systems (ICCCAS), Guilin, China, June 2006, pp. 1031-1034

[24] Z. Chen, J. Yuan, and B. Vucetic, "Analysis of transmit antenna selection/maximal-ratio combining in Rayleigh fading channels," IEEE Trans. Vehic. Tecnology, vol. 54, no. 4, pp. 1312-1321, July 2005.

[25] J. W. Craig, "A new, simple and exact result for calculating the probability of error for two-dimensional signal constellations," in Proc. IEEE Military Commun. Conf. (MILCOM), Boston, MA, November 1991, pp. 571-575.

[26] M. R. Spiegel and J. Liu, Mathematical Handbook of Formulas and Tables. 2nd edition: McGraw-Hill, 1999.

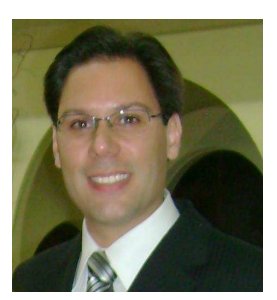

Renato Machado ( $\left.\mathrm{S}^{\prime} 04-\mathrm{M}^{\prime} 08\right)$ was born in Jaú, SP, Brazil, in 1979. He received the B.S. degree in electrical engineering from the São Paulo State University (UNESP), Ilha Solteira, SP, Brazil, in 2001. He received the M.Sc. degree and the Ph.D. degree in electrical engineering from the Federal University of Santa Catarina (UFSC), Florianópolis, SC, Brazil, in 2004 and 2008, respectively. He was a Visiting Researcher in the Department of Electrical Engineering, Arizona State University (ASU), Tempe, AZ, U.S.A., from August 2006 to June 2007. $\mathrm{He}$ was a Research Engineer in the Nokia Institute of Technology, Brazil, from October 2007 to March 2008. He was a Visiting Professor in the Department of Electrical Engineering, Federal University of Juiz de Fora, MG, Brazil, from September 2008 to August 2009. Since August 2009 he has been an Assistant Professor in the Federal University of Santa Maria, RS, Brazil. His research interests include MIMO systems, space-time coding, linear dispersion codes, cooperative diversity, wireless communications, and power line communications.

Dr. Machado is a member of the IEEE Communications Society, and the Brazilian Telecommunications Society.

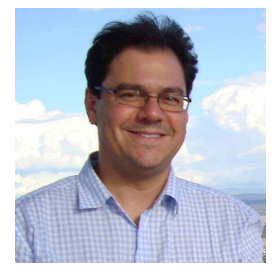

Bartolomeu F. Uchôa-Filho (S'94-M'96) was born in Recife, Brazil, in 1965. He received the B.S.E.E. degree from the Federal University of Pernambuco (UFPE), Recife, Brazil, in 1989; the M.S.E.E. degree from the State University of Campinas (UNICAMP), Campinas, Brazil, in 1992; and the Ph.D. degree in electrical engineering from the University of Notre Dame, Notre Dame, Indiana, U.S.A., in 1996. During 1997-1999 he held a Post-Doctoral position at the State University of Campinas. From August 1999 to January 2000 he was a Visiting Researcher in the Department of Electrical Engineering, Federal University of Santa Catarina, Florianópolis, Brazil. Since February 2000 he has been an Associate Professor in the same Department. From March 2009 to February 2010, he was a PostDoctoral Fellow at the University of Sydney, Australia. His research interests are in the area of coding and information theory, with applications to digital communications systems.

Dr. Uchôa-Filho is a member of Eta Kappa Nu, the IEEE Information Theory Society, and the Brazilian Telecommunications Society. For the 20032004 period, he served as the Editor-in-Chief of the Journal of the Brazilian Telecommunications Society. He was the Technical Program Committee Chair of the 27th Brazilian Telecommunications Symposium (SBrT'09).

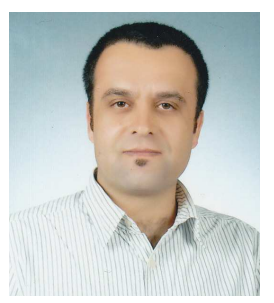

Tolga M. Duman received the B.S. degree from Bilkent University in 1993, M.S. and Ph.D. degrees from Northeastern University, Boston, in 1995 and 1998, respectively, all in electrical engineering. Since August 1998, he has been with the School of Electrical, Computer and Energy Engineering of Arizona State University where he is currently a full professor. Dr. Duman's current research interests are in digital communications, wireless and mobile communications, channel coding, turbo codes, coding for recording channels, and coding for wireless communications. He is the co-author of a textbook "Coding for MIMO Communication Systems" published by Wiley (2007).

Dr. Duman is the recipient of the National Science Foundation CAREER Award and IEEE Third Millennium medal. He has served as an editor for IEEE Trans. on Wireless Communications from 2003-2008, and he is currently an editor for IEEE Trans. on Communications. 